\title{
EL MUSEO "LA CASA ENCANTADA" DE BRIONES EN LA ENSEÑANZA DE LA HISTORIA: PROPUESTA PARA UNA SALIDA ESCOLAR PARA EDUCACIÓN PRIMARIA
}

\author{
Sebastián Molina Puche \\ Universidad de Murcia
}

\begin{abstract}
RESUMEN: Como ha sido de sobra demostrado, para que las salidas escolares desplieguen todo su potencial didáctico (esto es, para que sirvan para estimular el aprendizaje por "descubrimiento guiado"), han de estar enmarcadas en itinerarios didácticos y con ello, ser consideradas como parte integrante de la programación didáctica del curso. Esa integración curricular de las salidas es uno de los principales problemas con los que se encuentra el docente a la hora de poner en práctica este importante recurso didáctico. Con este trabajo pretendemos, además de mostrar las muchas posibilidades que tiene el museo de "La casa encantada" de Briones (sección etnología del Museo de La Rioja) para la enseñanza de la historia en Educación Primaria, indicar cómo pueden integrarse curricularmente las visitas al mismo en cada uno de los tres ciclos que conforman esa etapa educativa.
\end{abstract}

ABSTRACT: It has been demonstrated, in order that the school excursions develop all his didactic potential (this is, in order that they serve to stimulate the "guided discovery" learning), have to be framed in didactic itineraries and with it, to be considered as an integral part of the didactic programming of the course. The curricular integration of the school excursions is one of the principal problems which the teacher meets at the moment of putting in practical this important didactic resource. With this work we claim, beside showing many possibilities that there has the museum of "The Enchanted House" of Briones (section ethnology of the Museum of La Rioja) for the learning of the history in Primary Education, to indicate how they can integrate in the curriculum the visits to the museum in each of three cycles that shape this educational level.

PALABRAS CLAVE: Salidas escolares, didáctica de la Historia, Educación Primaria.

KEYWORDS: School excursions, didactics of the History, Primary Education. 


\section{Salidas escolares e itinerarios didácticos}

No cabe duda que las salidas escolares tienen una importancia de primer orden en el proceso de enseñanza-aprendizaje de todo alumno: sólo por medio de éstas se puede, parafraseando a Araceli Vilarrasa (2003), "salir del aula para reapropiarse del contexto", sólo las salidas permiten al alumno alcanzar una serie de vivencias propias, de experiencias directas, que difícilmente pueden obtener entre las cuatro paredes del aula.

Independientemente de la denominación elegida -como ha indicado Gabriel Travé (2004: 43), este recurso didáctico ha sido calificado como paseo escolar, visita educativa, excursión extraescolar, y más recientemente, como salidas de investigación, que es la que utiliza este autor para acentuar el carácter indagatorio de las mismas-, la gran aportación de las actividades realizadas extramuros del recinto escolar es que permiten al alumnado obtener una serie de conocimientos por medio de la observación, la vivencia, la manipulación e incluso la comprobación directa que, además, pueden facilitar la ampliación de destrezas específicas y promover su desarrollo personal (Wass, 1992: 14).

Evidentemente, esta apertura al mundo, esta posibilidad de observar y analizar in situ toda una serie de elementos diversos, es importante en todas las áreas de experiencia (nos referimos en este caso a la etapa de Educación Infantil) y conocimiento (etapa de Educación Primaria). No obstante, y por la naturaleza, finalidades, objetivos y contenidos que se tratan, es en el área de Conocimiento del Medio Natural, Social y Cultural (y en el caso de Educación Infantil, de Conocimiento del Entorno), donde esta observación directa es, si cabe, aún más importante.

En efecto, en un área cuya finalidad última es mostrar al alumnado el medio (en su sentido más amplio: un medio holístico, de carácter interactivo, espacialmente diferenciado, de experiencias multidimensionales, proyectivo y cambiante: Vilarrasa, 2002) en el que vive, ese "...conjunto de factores, fenómenos y sucesos de índole diversa y variada que configuran el contexto en el que tienen lugar las actuaciones de las personas y con relación a ellas dichas actuaciones adquieren un significado" (MEC, 1991), la posibilidad de trabar contacto directo con muchos de los citados factores, fenómenos y sucesos, demuestra tener una importancia clave.

Sin embargo, y como muy acertadamente indicó hace unos años Antonio L. García Ruiz (1994: 117) en un interesante trabajo sobre los itinerarios didácticos aplicados a la enseñanza de la Geografía (y quisiera subrayar el hecho de hablar, en este caso, de itinerarios didácticos, y no de salidas escolares, a fin de marcar la diferencia entre ambos, que más tarde trataré de explicar), la consideración general de los citados itinerarios ha sido siempre de segundo nivel: las clases y aprendizajes importantes son los que se dan en el aula, y todo aquello que se realice fuera de ella no es más que un complemento en muchos casos prescindible. Para este autor la razón explicativa de esta, por denominarla de alguna manera, "Ilamativa contradicción" entre la importancia que teóricamente debieran tener estas actividades de observación y experimentación fuera del aula para las áreas y asignaturas de Ciencias Sociales, y la insuficiente valoración y comprensión de las mismas radica, en buena parte, en la falta de información teórica sobre el uso lógi- 
co de este recurso didáctico que, en muchos casos, es a su vez la causante de que las experiencias realizadas no sean satisfactorias ni para el docente que las pone en práctica ni para el alumnado al que van dirigidas.

El problema reside en el hecho de que, en muchos casos, y tal vez debido a la citada falta de información teórica -si bien es cierto que contamos con trabajos en los que se ofrecen propuestas de sistematización de las salidas escolares desde fechas relativamente tempranas, como los de García y Martínez, 1988, y Liceras, 1992-, las actividades que se realizan fuera del recinto escolar no suelen ser objeto de la debida y necesaria preparación didáctica, y no es nada extraño que, además, no lleguen a plantearse como parte integrante de la programación didáctica del curso, lo que convierte a la visita o salida escolar en un elemento externo, e incluso ajeno, al resto de la materia impartida. En estas actividades poco organizadas o que no quedan claramente relacionadas con el resto del currículo impartido, el alumno observa y experimenta durante la propia salida, pero en muchos casos esas vivencias y observaciones carecen tanto de una explicación previa, como de una continuidad y un seguimiento en el aula, lo que las convierte en unas experiencias, cuanto menos, inapropiadas, en tanto que, aunque se estimula el aprendizaje por descubrimiento directo, no se logra poner en práctica lo que Pilar Benejam (2003: 10) ha denominado como "método por descubrimiento guiado".

En efecto, como ha señalado esta autora, no cabe duda que en todos los niveles educativos, pero en especial en los más tempranos o iniciales (nos referimos, evidentemente, a las etapas de Educación Infantil y Educación Primaria), el aprendizaje por descubrimiento directo, basado en el principio de la observación y la experimentación personal directa, aunque en su concepción teórica pueda parecer muy válido, resulta a todas luces insuficiente y poco recomendable para el alumnado, en tanto que el niño, cuando observa, ve únicamente lo que ya sabe, lo que quiere ver o lo que tiene sentido para él. Todo ello lleva a que sea preciso guiar esa mirada para que pueda alcanzar nuevos conocimientos, para que pueda comprender las causas y consecuencias de lo observado, y sepa interpretarlo y valorarlo. Debido a ello, resulta mucho más efectivo poner en práctica el citado "método por descubrimiento guiado", en el cual se incita al alumno a reflexionar sobre la realidad, pero bajo la tutela y dirección del profesor, que es quien decide qué observar, qué se ha de comprobar, y sobre qué hay que recapacitar, así como las pautas a seguir en cada una de las tareas. Un método que aúna la actividad del alumnado, la significación y la construcción de los conocimientos (Benejam, 2003: 11), y para el cual hay que hacer uso, evidentemente, de salidas escolares pero no como actividades aisladas del resto del currículo impartido y de carácter excepcional, sino integradas en esos hilos conductores, esos instrumentos de planificación que permiten al profesorado organizar esas salidas, poner en contacto al alumnado con el medio y articular un proceso de enseñanza-aprendizaje, que son los itinerarios didácticos (Vilarrasa, 2002).

La salida, la excursión o visita se convierte, de esta manera, no en un fin en sí misma, sino en parte de un programa didáctico (el itinerario) que la integra y le otorga sentido y significación. Ya no se trata sólo de ver, sentir, observar, sino que esos elementos a observar, a manipular o a experimentar se ubican dentro de un marco 
educativo adecuado en el cual la experiencia directa, vivida a partir de la interacción espontánea con el entorno y la experimentación científica, van de la mano (Vilarrasa, 2003).

Así, la salida se convierte en una fase, una etapa de un proceso didáctico más amplio y complejo que la integra: si en una primera fase se fundamenta y prepara la visita explorando las ideas del alumnado sobre la temática, al tiempo que se planifica el trabajo y los recursos disponibles, la salida supondría una segunda etapa donde se habrían de materializar las tareas propuestas; y por último, se asistiría a una etapa posterior en la cual se habría de organizar, clasificar y ordenar el material obtenido en la fase anterior, y se confeccionaría el pertinente informe de trabajo que recogiera las conclusiones a las que se hubiera llegado (Travé, 2004: 44).

De esta manera, las salidas, integradas en itinerarios didácticos -que, a su vez, se encuentran integrados en el currículo, generalmente formando parte de una unidad didáctica: Vilarrasa, 2002-, sirven sobre todo para que el alumnado tenga la oportunidad de percibir y analizar sobre el terreno (experimentar, en su doble acepción), aquello que está aprendiendo a nivel teórico en el aula, uniendo de esta manera, como propugna Benejam, los conocimientos transmitidos -obtenidos en el aula- y aquellos procedentes de la investigación y el descubrimiento propio -alcanzados en la fase del itinerario en la que se realiza la salida escolar-.

En otras palabras, las salidas escolares integradas en itinerarios didácticos $-y$ por ende, como componentes "de pleno derecho" de una programación didáctica- facilitan, por un lado, que las actividades realizadas fuera del recinto escolar no se tengan que subordinar al trabajo en el aula, ya que, al considerarse una parte importante del proceso didáctico, ya no se va a tratar de un mero complemento de todos aquellos contenidos que se han impartido en el aula, sino que una importante parcela de esos contenidos van a ser tratados directamente durante la salida. Y por otro lado, dicha integración de las salidas en itinerarios didácticos permite que desaparezca esa faceta de exclusión y alejamiento del currículo que, en muchos casos, tienen -o parecen tener- las citadas salidas escolares.

La necesidad de preparar la visita con antelación, de realizar actividades en el aula para motivar al alumnado, movilizar los conocimientos previos que éstos tienen sobre la temática a trabajar o sobre el lugar donde va a realizarse las labores de observación directa o experimentación, así como la necesidad de preparar actividades a realizar durante la salida, y actividades de utilización de los datos obtenidos durante la misma a modo de conclusión, provocan que poco o nada quede para la improvisación (del largo listado de tareas a realizar antes, durante y después de una salida escolar podemos encontrar un buen ejemplo en Urones y Sánchez, 1997) y, sobre todo, que de esta manera la salida quede necesariamente incluida en un programa de trabajo, una planificación didáctica que impida que aquella quede desgajada o excluida del currículo impartido en el aula. En este sentido, ya señalaban Antonio L. García y José M. Martínez en su artículo de 1988 que, como toda actividad docente, las salidas deben estar planificadas desde el comienzo del curso, una planificación que debe ir en consonancia con la planificación general del curso y de la materia buscando siempre la conexión entre lo estudiado en el aula y fuera de 
ella, entre la teoría y la práctica, porque ambas son necesarias en todos los niveles de la enseñanza (García y Martínez, 1988: 110).

Al contrario de lo que pudiera parecer, la integración curricular de itinerarios y salidas, y la consiguiente planificación de las mismas desde comienzos del curso no es, en modo alguno, un hecho excesivamente complejo: no debe haber problema para aquellas salidas que, por realizarse en ciertos lugares "a cubierto" (edificios religiosos o civiles, monumentos, museos), pueden proyectarse sin temor a los "factores temporales y estacionales" de los que nos hablaba Stephen Wass (1992: 47); pero tampoco para el resto, si se diseñan con la suficiente flexibilidad de fechas para su realización. De manera que, en principio, y únicamente teniendo en cuenta una serie de factores básicos -festividades, horarios de visita, posibilidad de retrasar o adelantar la actividad fuera del aula sin que suponga un perjuicio para la consecución de los objetivos previstos-, nada impide que desde comienzos de curso se puedan planificar visitas y salidas destinadas a completar, aumentar o profundizar conocimientos de tipo geográfico, histórico, etnológico o artístico mediante la visita a museos $u$ otros lugares de interés.

En síntesis, no cabe duda que si lo que se pretende es que las actividades realizadas fuera del aula dispongan de una entidad y un peso específico suficientes en el conjunto de acciones desarrolladas para impartir los contenidos previstos en las unidades didácticas donde queden integradas -o lo que es lo mismo, que las salidas aporten enseñanzas significativas y útiles para el alumnado-, se debe llevar a cabo una labor de integración curricular de esas salidas escolares.

Evidentemente, esto supone que la primera tarea a realizar sea la de precisar cómo se puede incluir el lugar a visitar, y todo lo que éste nos ofrece, en la programación anual, o lo que es lo mismo: saber relacionar lo que se va a observar durante la salida con una temática concreta, a fin de que haya una correlación directa entre lo tratado en el aula y el lugar visitado. Es decir -y con ello, una obviedad aparente-: es algo tan sencillo como, por ejemplo, visitar y utilizar todo lo que nos ofrece el yacimiento de Velilla de Aracanta (cuyas posibilidades didácticas han sido estudiadas por García y Castillo, 2006) en el transcurso de la unidad didáctica en la que se desarrollen contenidos relacionados con la romanización o la Edad Antigua; visitar la iglesia de Santa María de Palacio de Logroño en el desarrollo de una unidad didáctica en la que se trate el arte gótico, el camino de Santiago o la Edad Media; o el museo Histórico-Arqueológico de Nájera en aquellas otras en las que, por ejemplo, se pretendan tratar aspectos básicos del tiempo histórico como son el cambio y la continuidad (algo perfectamente observable en un museo de estas características, en el que se ofrece un paseo panorámico por la historia de la localidad).

Sin embargo, lo que en algunos casos parece evidente (uso de Contrebia Leukade para la cultura celtíbera, de Velilla de Aracanta, el yacimiento de La Clínica o el museo de Calahorra para la romanización, de Santa María de Palacio, el monasterio de Cañas o la catedral de Santo Domingo de La Calzada para el arte gótico o la Edad Media...), en otros, como pueda ser el recientemente abierto museo de "La casa encantada" de Briones, en la cual se recrea lo que podría ser la casa-tipo tradicional riojana, esa ubicación curricular de la visita no parece tan obvia, algo que podría frenar al docente a la hora de seleccionarla como objeto de una salida esco- 
lar. Evidentemente, se trata de una dificultad sólo aparente: son muchas las posibilidades que ofrece un museo de esas características para la enseñanza de la Historia en las distintas etapas educativas, y en particular en Primaria, como podemos ver a continuación.

\section{2. "La CaSa encantada" De Briones para la enseñanZa de la historia en Educación Primaria}

Tal vez resulte ocioso tratar de defender la importancia que actualmente se otorga a los museos como herramienta educativa (Asensio y Pol, 2003): los museos, en tanto que contenedores de patrimonio y cultura (Fernández, 2003: 59), conforman el principal lugar de referencia en lo que a visitas escolares se trata (buen ejemplo de ello podemos encontrarlo en Cataluña, donde las visitas a museos ocupan gran parte de las actividades programadas por los centros de enseñanza: Fernández, 2003) gracias, sobre todo, a los considerables esfuerzos que últimamente se están realizando en muchos de ellos para dotarles de una faceta didáctica y pedagógica que anteriormente muy pocos tenían. De hecho, actualmente son pocas las entidades museísticas que carecen de un taller didáctico permanente o en las que no se organicen habitualmente talleres temporales destinados a la enseñanza del "público infantil": sirva como ejemplo el aula didáctica existente en el monasterio de Yuso, o los organizados por la propia Casa de las Ciencias de Logroño. De manera que consideramos que no es necesario mostrar lo mucho que pueden aportar los museos en general, y el que nos ocupa en particular, para el aprendizaje del alumnado, y particularmente en las áreas y asignaturas correspondientes a las Ciencias Sociales, pues existen multitud de trabajos en que han mostrado ese extremo: por citar algunos ejemplos recientes, son buenos referentes de ello los artículos recogidos en el número monográfico que la revista íber dedicó en 2004 a museos y didáctica de las ciencias sociales, y los trabajos publicados en el también monográfico Museo y patrimonio en la didáctica de las ciencias sociales publicado por la Universidad de Huelva en 2001.

En este sentido, consideramos mucho más efectivo y edificante no tanto mostrar lo importante que puede resultar la realización de una visita al museo de "La casa encantada" para la enseñanza de la historia -un extremo que damos por hecho-, sino cómo se pueden integrar curricularmente esas salidas en la etapa de Educación Primaria.

Comencemos por describir qué nos ofrece el museo.

Abierta al público en agosto del año 2007, "La casa encantada" de Briones es un museo etnográfico (de hecho, se trata de la sección de Etnología del Museo de La Rioja) ubicado en el palacio del marqués de San Nicolás, un edificio barroco de 1755 situado en el centro de la población (Sánchez Trujillo, 2008). Distribuido en cuatro pisos, que ocupan nada menos que 800 metros cuadrados, el museo recrea una casa tradicional riojana de tres plantas (la planta baja está dedicada a introducir y explicar al visitante lo que va a encontrar en la exposición permanente) en la que se conservan y exhiben medio millar de piezas originales de los siglos XVIII al XX.

La primera planta acoge la cocina (donde destacan los utensilios para la elaboración del pan, el queso y los embutidos) y la despensa donde se almacenaban los alimentos, así como el comedor y una sala de estar en las que se que recrea lo que 
podría considerarse como parte noble, el espacio público de la casa tradicional riojana. Frente a ese espacio público del comedor, en la segunda planta se recrea el área más privada de la casa: los cuartos de labores domésticas, de higiene personal y los dormitorios. En esta segunda planta podemos encontrar una nutrida representación de útiles para la elaboración de tejidos (hiladoras, telares, costura), para la limpieza doméstica, de lavado y planchado de ropa..., así como de aseo personal, que generalmente se realizaba en la intimidad del dormitorio. Así, en los espacios en los que se recrean los dormitorios de niños y adultos, podemos encontrar, además de camas, cunas, andadores, cochecitos de bebés y una amplia muestra de juguetes, también enseres de aseo personal y belleza. La tercera y última planta (actualmente en proceso de preparación y montaje) se va a dedicar al ocio, a los juegos y fiestas tradicionales de La Rioja, a la celebración religiosa y devocional, pero también a la diversión, la música y el baile.

Es decir, "La casa encantada" de Briones, como todo museo etnográfico de estas características, exhibe toda una serie de enseres, herramientas y utensilios domésticos de otras épocas que lo convierten en un interesantísimo y muy útil instrumento didáctico para la enseñanza de la historia en las distintas etapas educativas, pero sobre todo para la etapa de Educación Primaria, porque permite mostrar, por medio de elementos fácilmente reconocibles e identificables, tanto el paso del tiempo, como los cambios y permanencias que se producen a lo largo de éste, sin necesidad de hacer demasiadas referencias cronológicas.

No obstante, y aunque en esta ocasión vamos a centrarnos en las posibles aplicaciones que puede tener la visita a este museo para la etapa de Educación Primaria (y más concretamente, para la parte social y cultural del área de Conocimiento del Medio), somos muy conscientes de que también en Educación Infantil (no es nada inusual el uso de visitas a museos en esta etapa, como demuestran Bodes, Sueiras y Tejerina, 1997) y Educación Secundaria Obligatoria puede hacerse uso de este museo para ilustrar o impartir conocimientos de carácter histórico a través de lo que allí se conserva.

Es más, si tenemos en cuenta la reducida oferta que actualmente existe en la Comunidad Autónoma de La Rioja en lo que se refiere a museos de carácter arqueológico-histórico, la importancia de "La casa encantada" de Briones como herramienta para la enseñanza de la historia en las distintas etapas educativas aumenta significativamente. En efecto, como es bien sabido, el Museo de La Rioja (museo arqueológico provincial con sede en Logroño) se encuentra cerrado, desde hace años, a la espera de unas reformas que no llegan a ultimarse; y el Museo Diocesano de Calahorra se encuentra, como toda la catedral, igualmente cerrado por obras. Lo que convierte a "La casa encantada" de Briones, junto al museo municipal de Nájera y el recientemente reabierto Museo Municipal de Calahorra -una auténtica referencia para comprender la romanización en la zona-, en los únicos que actualmente están disponibles para ilustrar la historia de la región.

Pero volvamos a lo que puede ofrecer este museo para las etapas de Educación Infantil y Secundaria Obligatoria.

Una visita a "La casa encantada" puede ser de gran utilidad para que el alumnado de segundo ciclo de Educación Infantil pueda aprender las temáticas que, en el tercer 
bloque de contenidos del área de Conocimiento del Entorno, titulado "Cultura y vida en sociedad", hacen referencia a las señas de identidad regional (no en vano el museo recrea una casa tradicional típica riojana y en él aparecen referencias al arte popular, al folclore y las tradiciones de la tierra), y al reconocimiento del paso del tiempo (en tanto que el alumno puede observar claramente los cambios que se han producido en elementos cotidianos que puede reconocer en su propio entorno doméstico).

Cuadro 1. Contenidos propios de las ciencias sociales del área de Conocimiento del Entorno.

\section{Bloque 3. Cultura y vida en sociedad}

- La familia y la escuela como primeros grupos sociales de pertenencia. Toma de conciencia de la necesidad de su existencia y funcionamiento mediante ejemplos del papel que desempeñan en su vida cotidiana. Valoración de las relaciones afectivas que en ellos se establecen.

- Observación de necesidades, ocupaciones y servicios en la vida de la comunidad.

- Incorporación progresiva de pautas adecuadas de comportamiento, disposición para compartir y para resolver conflictos cotidianos mediante el diálogo de forma progresivamente autónoma, atendiendo especialmente a la relación equilibrada entre niños y niñas.

- Reconocimiento de algunas señas de identidad cultural del entorno e interés por participar en actividades sociales y culturales. Reconocimiento de la bandera, el escudo y el himno de La Rioja. Identificación del Parlamento y de los edificios más emblemáticos de la cultura, la historia y el arte en La Rioja.

- Identificación de algunos cambios en el modo de vida y las costumbres en relación con el paso del tiempo.

- Interés y disposición favorable para entablar relaciones respetuosas, afectivas y recíprocas con niños y niñas de otras culturas.

Fuente: CAR, 2007a.

De igual modo, tanto en segundo como en cuarto curso de ESO, la visita a este museo puede servir para ilustrar todo tema en el que se trate sobre la vida cotidiana en la Europa preindustrial, caso, por ejemplo, de "La forma de vida en las ciudades cristianas" (contenido perteneciente al bloque III: Las sociedades preindustriales, de segundo curso de ESO), o "La nueva sociedad del siglo XIX: viejas y nuevas elites; las clases populares" (contenido perteneciente al bloque II: Bases históricas de la sociedad actual, de cuarto curso de ESO: CAR, 2007c).

Ya centrados en el caso particular de la etapa de Educación Primaria, la utilidad que "La casa encantada" de Briones puede tener para esta etapa educativa, arranca del hecho de que una visita a la misma puede contribuir de manera muy favorable a alcanzar uno de los objetivos que el actual decreto de currículo (CAR, 2007b) contempla para el conjunto de esta etapa, nos referimos al objetivo: 
“m) Conocer el patrimonio cultural de España, participar en su conservación y mejora y respetar y valorar como un bien enriquecedor para todos los españoles su diversidad lingüística y cultural".

Es más, convertir toda una serie de objetos de uso cotidiano, en modo alguno llamativos o excepcionales (no se trata de grandes obras de arte, ni piezas únicas e irreemplazables), en piezas de museo, puede ser un auténtico acicate para que estos alumnos puedan comprender que el patrimonio -en este caso, en su faceta culturales algo que les afecta directamente, pues incluye también elementos que pueden tener a su alcance, y por tanto, que su conservación y valoración también les corresponde a ellos.

De igual modo, aunque de forma mucho más concreta, la realización de una visita a "La casa encantada" desarrollada desde el ámbito del área de Conocimiento del Medio puede contribuir a que se alcancen tres de los objetivos propios de esa área de conocimiento, como son (CAR, 2007b):

"7. Identificar los principales elementos del entorno natural, social y cultural, resaltando los de La Rioja, analizando su organización, sus características e interacciones y progresando en el dominio de ámbitos espaciales cada vez más complejos".

"8. Reconocer en el medio natural, social y cultural, cambios y transformaciones relacionados con el paso del tiempo e indagar algunas relaciones de simultaneidad y sucesión para aplicar estos conocimientos a la comprensión de otros momentos históricos, tomando algunos ejemplos de La Rioja".

"9. Conocer y valorar el patrimonio natural, histórico y cultural de España y de La Rioja, respetando su diversidad y desarrollando la sensibilidad artística y el interés por colaborar activamente en su conservación y mejora".

No cabe duda que conocer la composición y elementos de la casa tradicional riojana puede facilitar la identificación de uno de los elementos del entorno social y cultural de esta región, así como reconocer, por medio de un ejemplo cercano, los cambios, transformaciones y permanencias relacionadas con el paso del tiempo, y aprender a valorar el patrimonio histórico y cultural de La Rioja y España.

Con todo, y como hemos señalado anteriormente, uno de los principales problemas con los que se puede encontrar el docente de Educación Primaria a la hora de diseñar un itinerario didáctico en el que se lleve a cabo una visita al citado museo etnológico de Briones es la integración de itinerario y salida en la programación de aula: en principio, no parece sencillo relacionar una visita a un museo en el que se exhiben enseres domésticos con las temáticas comprendidas en los bloques de contenidos del área en sus distintos ciclos, pues sólo en el segundo de ellos se contempla el tratamiento de la vida cotidiana en el pasado. Evidentemente, no es así en modo alguno: en cualquiera de los tres ciclos que forman parte de esta etapa tiene perfectamente cabida una visita a un museo de estas características.

En efecto, las temáticas incluidas en el quinto bloque de contenidos, el titulado "Historia. El cambio en el tiempo" (CAR, 2007b) permiten relacionar perfectamente los conocimientos que pueden alcanzarse por medio de la observación de los elementos conservados en el citado museo con las enseñanzas que se puedan impartir en el aula.

Así ocurre, en el caso del Primer ciclo, en las unidades didácticas en las que se traten los contenidos del quinto bloque que Ilevan por título "La riqueza histórica 
del entorno próximo. Construcciones civiles y religiosas. Cuidado y conservación" y "Aproximación a la Historia: algunos acontecimientos relevantes del pasado". Si para la primera los enseres domésticos de "La casa encantada" pueden servir, entre otras cosas, para mostrar la riqueza histórica que se atesora en muchos hogares riojanos, y la necesidad de conservarlos; en el caso de la segunda temática una visita a este museo puede servir para mostrar, cuanto menos, evidencias del paso del tiempo y manifestaciones del pasado, es decir, dos de las variables principales del concepto de tiempo histórico (cambio y permanencia) que se han de impartir en ese ciclo, por ser las más acordes a la edad de los alumnos y su capacidad de comprensión del concepto (Trepat y Comes, 1998).

En el Segundo ciclo, la visita a "La casa encantada" puede llevarse a cabo a lo largo del desarrollo de las unidades didácticas en las que se traten los contenidos "Cambios y permanencias. Acontecimientos históricos y vida cotidiana. Testimonios y herencias del pasado", y "La riqueza artística de La Rioja. Reconocimiento y valoración del patrimonio artístico". No cabe duda que lo conservado y exhibido en el museo permite mostrar los cambios y permanencias que pueden observarse en la vida cotidiana, así como los testimonios y herencias del pasado. Pero también es una buena muestra de la riqueza artística de La Rioja, pues al fin y al cabo, esos enseres son un ejemplo del arte y la cultura popular riojana.

Por último, en el Tercer ciclo de esta etapa, en el cual en el quinto bloque de contenidos se han de tratar "Aspectos básicos de la Historia de España y, de manera particular, de La Rioja", los elementos conservados en el museo pueden ser un buen ejemplo para intentar ilustrar la vida en La Rioja durante la Edad Moderna y hasta bien entrado el siglo XX, así como para abordar los contenidos "Conocimiento, valoración y respeto por manifestaciones culturales y artísticas más relevantes del patrimonio histórico y cultural. El patrimonio cultural y artístico de La Rioja: manifestaciones más relevantes, museos".

En síntesis, no cabe duda que el actual currículo de Educación Primaria de la Comunidad Autónoma de La Rioja permite perfectamente que el docente pueda diseñar con antelación (y lógicamente, integrar sin problema alguno en su programación anual de aula) visitas a entidades museísticas como la que nos ocupa: como hemos mostrado, pese a que aparentemente pueda considerarse difícil, los contenidos previstos en el actual Decreto de Currículo de la CAR facilita que sean muchas las ocasiones para hallar una correlación temática directa entre lo que se ha de impartir en el aula, y lo que se puede descubrir, observar y analizar en este museo.

No cabe duda que la ubicación curricular y la correlación temática son las que deben guiar toda salida escolar, lo que supone, a su vez, que la salida quede integrada en una secuencia didáctica (el itinerario) que convierta la visita en una fase de un proceso educativo más amplio. Sólo de esta manera los conocimientos adquiridos pueden ser lo suficientemente significativos como para que el alumno, además de interiorizarlos, guarde recuerdo (en definitiva, aprenda) de ellos. Y en este sentido, como hemos señalado, no existe problema alguno, en hallar ubicación curricular a las salidas escolares que se puedan realizar a "La casa encantada" de Briones, sección etnología del Museo de La Rioja, en el ámbito de las ciencias sociales en los tres ciclos de Educación Primaria. 


\section{REFERENCIAS BIBLIOGRÁFICAS}

ASENSIO, M. y POL, E. (2003). Aprender en el museo. Íber: Didáctica de las ciencias sociales, geografía e historia, 36, 62-77.

ÁVILA, R. M. (2003). La función de los itinerarios en la enseñanza y el aprendizaje del patrimonio histórico-artístico. Una reflexión didáctica. Íber: Didáctica de las ciencias sociales, geografía e historia, 36, 36-46.

BENEJAM, P. (2003). Los objetivos de las salidas. Iber: Didáctica de las ciencias sociales, geografía e historia, 36, 7-12.

BODES, M. J.; SUEIRAS, M. E. y TEJERINAS, E. A. (1997). Un día en la mina. Cuadernos de Pedagogía, 256, 17-19.

CAR (2007). Decreto 25/2007, de 4 de mayo, por el que se establece el Currículo del Segundo Ciclo de Educación Infantil en la Comunidad Autónoma de La Rioja (BOLR 8 mayo).

CAR (2007b). Corrección de errores del Decreto 26/2007, de 4 de mayo, por el que se establece el currículo de la Educación Primaria en la Comunidad Autónoma de La Rioja (BOLR 2 de junio).

CAR (2007c). Decreto 23/2007, de 27 de abril, por el que se establece el Currículo de la Educación Secundaria Obligatoria de la Comunidad Autónoma de La Rioja (BOLR 3 mayo).

DELGADO, E. y ALARIO, M. T. (1994). La interacción fuera del aula: itinerarios, salidas y paseos. Tabanque: Revista pedagógica, 9, 155-178.

FERNÁNDEZ CERVANTES, M. (2003). Los museos: espacios de cultura, espacios de aprendizaje. Íber: Didáctica de las ciencias sociales, geografía e historia, 36, 55-61.

GARCÍA RUIZ, A. L. (1994). Los itinerarios didácticos: una de las claves para la enseñanza y comprensión de la Geografía. Iber: Didáctica de las ciencias sociales, geografía e historia, 1, 117-126.

GARCÍA SANTAMARÍA, T. y CASTILLO PASCUAL, P. (2006). Velilla de Aracanta: una propuesta de talleres didácticos en un yacimiento arqueológico. Contextos educativos. Revista de Educación, 8-9, 295-314.

HERNÁNDEZ CARDONA, F. X. (2002). Didáctica de las ciencias sociales, geografía e historia. Barcelona: Graó.

INSA, Y. (2004). Los itinerarios didácticos. Aula historia social, 14, 87-91.

LICERAS, A. (1992). Los itinerarios didácticos y el trabajo de campo en la enseñanza de la geografía. Revista de educación de la Universidad de Granada, 6, 141-166.

MARTíNEZ LÓPEZ, J. M. y GARCÍA RUIZ, A. L. (1988). Los itinerarios didácticos en la enseñanza de las ciencias sociales. Revista de educación de la Universidad de Granada, 2, 107-114.

MEC (1991). Real Decreto 1006/91 de 14 de junio por el que se establecen las enseñanzas mínimas correspondientes a la Enseñanza Primaria (BOE 26 de junio).

MEDIR, R. M. (2003). Salir de la escuela: entre la tradición y la educación ambiental para la sostenibilidad. Íber: Didáctica de las ciencias sociales, geografía e historia, 36, 26-35. 
SÁNCHEZ TRUJILLANO, M. T. (2008). La Casa Encantada: Sección de etnología del Museo de la Rioja en el Palacio del Marqués de San Nicolás de Briones. Belezos: Revista de cultura popular y tradiciones de La Rioja, 8, 58-63.

TRAVÉ, G. (2003). Más allá de las paredes del aula: salidas de investigación escolar. Kikiriki. Cooperación educativa, 71-72, 43-46.

TREPAT C. A. y COMES, P. (1998). El tiempo y el espacio en la didáctica de las ciencias sociales. Barcelona: Graó.

URONES, C. y SÁNCHEZ-BARBUDO, M. C. (1997). La organización de salidas al entorno escolar. Revista Electrónica Interuniversitaria de formación del Profesorado, 1, 1-5.

VILARRASA, A. (2002). Las salidas escolares. Una estrategia para la integración curricular del estudio del medio local. Educación Primaria. Orientaciones y recursos (6-12 años). Barcelona: Praxis, pp. 48/1-48/20.

VILARRASA, A. (2003). Salir del aula. Reapropiarse del contexto. Iber: Didáctica de las ciencias sociales, geografía e historia, 36, 13-25.

VV. AA. (2001). Museo y patrimonio en la didáctica de las ciencias sociales. Huelva: Universidad de Huelva.

WASS, S. (1992). Salidas escolares y trabajo de campo en la educación primaria. Madrid: Morata. 